\title{
SELECTED PROBLEMS OF DAMAGE DETECTION IN INTERNALLY SUPPORTED PLATES USING ONE-DIMENSIONAL DISCRETE WAVELET TRANSFORM
}

\author{
Michae Guminiak, Anna Knitter-Piątkowska \\ Poznan University of Technology, Poznań, Poland \\ e-mail:michal.guminiak@put.poznan.pl; anna.knitter-piatkowska@put.poznan.pl
}

\begin{abstract}
The paper is concerned with damage detection in plates while using the Discrete Wavelet Transform (DWT). Rectangular plate structures resting on a Winkler or elastic half-space type foundation, with free boundaries are examined. Plate bending is described and solved by the Boundary Element Method in a direct approach. Defects are introduced by additional edges forming slots in relation to the basic plate domain. Numerical investigation is conducted basing on signal analysis of the structural static response and by taking advantage of multi-resolution analysis (MRA) of the signal function which can be represented in a multi-scale manner. The obtained signal is decomposed with the use of Daubechies or Coiflet wavelet families. The white noise generator is used to model measurement inaccuracy which is an inevitable element of a real experiment. The efficiency of DWT of the contaminated signal in damage detection is studied.
\end{abstract}

Keywords: Kirchhoff plates, Boundary Element Method, Discrete Wavelet Transform

\section{Introduction}

The aim of the present work is to detect the localization of defects provided that they exist in the considered plate structure. This problem has focused much attention and has been extensively investigated by many scientists. Some approaches are based on e.g. optimization of loads (Mróz and Garstecki, 2005), information on natural frequencies (Dems and Mróz, 2001), heat transfer (Ziopaja et al., 2011) or inverse analysis (Garbowski et al., 2011; Knitter-Piątkowska and Garbowski, 2013). Variety of methods are based on comparison of the response of damaged and undamaged structures. It is a serious difficulty since the experiments on these two structures must be carried out with identical boundary and loading conditions. Moreover, a global response of a structure is insensitive to the localized damage. Therefore, the uncertainty following from variation of the conditions in experiments can be larger than the precision of measuring gauges. A new impact came from a modern signal processing method, namely wavelet transformation (WT) (Wang and Deng, 1999) also in its discrete form (DWT) (Knitter-Piątkowska et al., 2014). WT can surprisingly well extract the desired detailed information from numerous data representing the global response of a damaged structure. Moreover, the information on the undamaged structure is not necessary.

Numerical investigation is carried out basing on signal analysis of the structural static response. Plate bending is described and solved by the Boundary Element Method in a direct form (Guminiak, 2016). The problem of damage detection in plates supported on the boundary and inside its domain was described in detail and illustrated e.g. in the paper (Knitter-Piątkowska et al., 2017). The boundary integral equations were derived in a singular and non-singular approach (Guminiak, 2016). There were considered rectangular plates resting on the half-space flexible foundation with bilateral constraints and on the Winkler-type flexible supports with unilateral 
(with the possibility of disconnection of the plate and the foundation) and bilateral (without such possibility) constraints. Defects in plates were modeled as slots near the plate boundary. As a structural response, vertical displacements or influence line of deflections erre taken into consideration. Decomposition of the obtained signal was carried out using DWT with Daubechies 4, Coiflet 6 and Coiflet 12 families of wavelets. Multiresolution signal analysis using Mallat pyramid algorithm was applied (Mallat, 1999).

Although the considered problem is two-dimensional from the point of view of deformation description, the applied one-dimensional DWT leads to efficient results in defect detection. Considered examples quite correctly identify the presence and position of damage. For the selected example, white noise has been introduced too.

\section{Theoretical consideration on wavelet transform}

In the presented paper, the wavelet transform will be implemented, in which for the representation of the signal $f(t)$ a linear combination of wavelet functions is used. Therefore, they are well-suited for dealing with signals having discontinuities. The theory of the wavelet transformation (WT) was presented in many papers e.g. (Dodge, 2003). Foundations of WT will be recalled below. First, let us consider the continuous wavelet transform of the signal $f(t)$ in the time and frequency domain

$$
W f(a, b)=\int_{-\infty}^{\infty} f(t) \bar{\psi}_{a, b}(t) d t
$$

where the overbar denotes the complex conjugate of the function under it. The function $\psi(t)$ is usually called the wavelet (mother) function and belongs to the field of $L^{2}(\mathbf{R})$. Additionally, the function $\psi(t)$ must satisfy the condition of admissibility (Mallat, 1999). This condition can be written in form of inequality

$$
\int_{0}^{\infty} \frac{1}{\omega}|\Psi(\omega)|^{2} d \omega<\infty
$$

The function $\Psi(\omega)$ can be treated as the Fourier transform of the function $\psi(t)$ :

$$
\Psi(\omega)=\int_{-\infty}^{\infty} \psi(t) \mathrm{e}^{-\mathrm{i} \omega t} d t
$$

The function $\Psi(\omega)$ is oscillatory and its average value is equal to zero, which leads to the equality

$$
\int_{-\infty}^{\infty} \psi(t) d t=0
$$

The mother wavelet may have real or complex-valued character. In the considered cases, realvalued wavelets will be applied. For signal decomposition, a set of wavelets (wavelet family) is applied. This set of functions is obtained by translating and scaling of the function $\psi$

$$
\psi_{a, b}=\frac{1}{\sqrt{|a|}} \psi\left(\frac{t-b}{a}\right)
$$

where $t$ denotes a time or space coordinate, $a$ is the scale parameter and $b$ the translation parameter. The parameters $a$ and $b$ take real values $(a, b \in \mathbf{R})$ and, additionally, $a \neq 0$. The 
element $1 / \sqrt{a}$ is the scale factor which ensures constant wavelet energy regardless of the scale. It means that the norm $\left\|\psi_{a, b}\right\|=\|\psi\|=1$. In the presented numerical solutions of plate bending problems the leading role takes the Discrete Wavelet Transform (DWT). According to this approach, the wavelet family can be obtained by substitution $a=1 / 2^{j}$ and $b=k / 2^{j}$ into equation (2.5). As a result, the following relation is obtained

$$
\psi_{j, k}(t)=\sqrt{2^{j}} \psi\left(2^{j} t-k\right)
$$

in which $k$ and $j$ are the scale and translation parameters, respectively. The meaning of these parameters for the simplest Haar wavelet is illustrated e.g. in papers (Guminiak, 2016; Dobrzycki and Mikulski, 2016).

Discrete signal decomposition can be written according to the Mallat pyramid algorithm in the known form (Mallat, 1999)

$$
f_{J}=S_{J}+D_{J}+\ldots+D_{n}+\ldots+D_{1} \quad n=J-j
$$

where each component in signal representation is associated with a specific range of frequency and provides information at the scale level $(j=1, \ldots, J)$. The discrete parameter $J$ describes the level of multi-resolution analysis (MRA), $S_{J}$ is a smooth signal representation, $D_{n}$ and $S_{n}$ are details and rough parts of the signal and $D_{1}$ corresponds with the most detailed representation of the signal. To fulfill the dyadic requirements of DWT, the function $f_{J}$ must be approximated by $N=2^{J}$ discrete values. The multi-resolution analysis according to the Mallat pyramid algorithm is illustrated in Fig. 1 and e.g. in papers (Mallat, 1999) and (Knitter-Piątkowska et al., 2017).

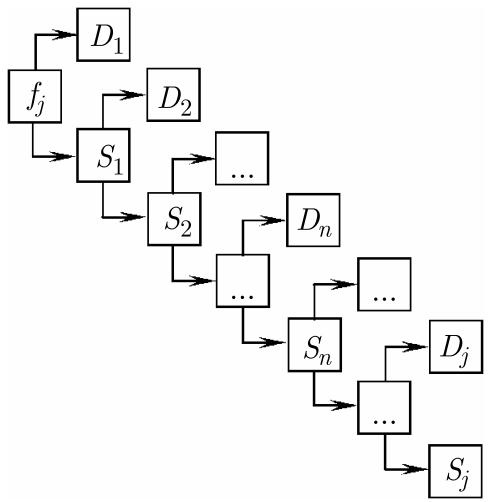

Fig. 1. Mallat pyramid algorithm (Mallat, 1999; Knitter-Piątkowska et al., 2017)

In the current analysis of defect detection, Daubechies and Coiflet wavelets will be applied. These family of wavelets are orthogonal, continuous and have a compact support. Daubechies wavelets are asymmetrical and Coiflet wavelets are nearly symmetric. Both families have sharp edges. These wavelet families do not require a large number of coefficients hence they are widely used in solving a broad range of problems e.g. image analysis or defect detection. The order of Daubechies wavelet family functions has the range between 2 and 20 and they are even numbers only. The Coiflet function accepts the even integers 6, 12, 18, 24 and 30. Daubechies wavelet of the second order corresponds to the simplest Haar wavelet. Basis and scaling functions of Daubechies 4 and Coilflet 6 wavelets are presented in Fig. 2.

\section{Simulation of measurement errors in numerical data}

Measurement (observational) error is the difference between the measured value of quantity and its true value (Dodge, 2003). Measurement errors are an inevitable element of any real experiment 
(a)

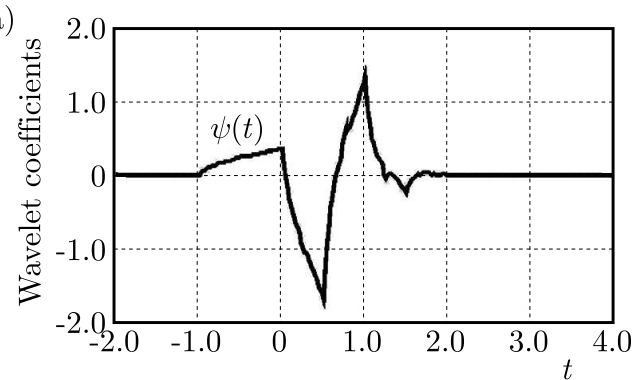

(c)

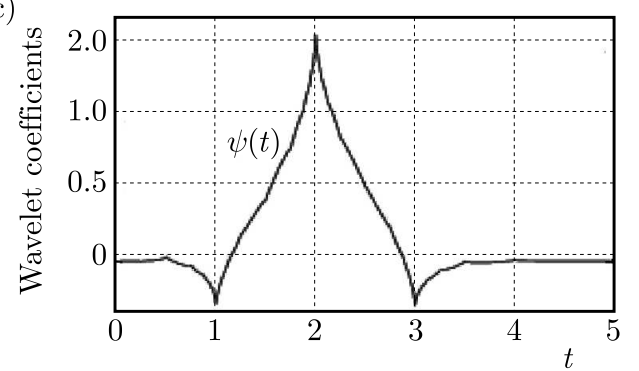

(b)

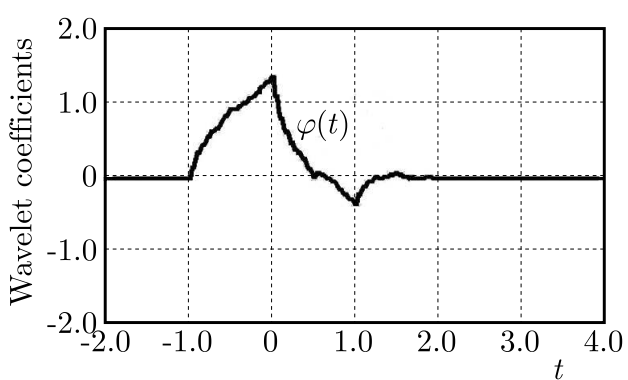

(d)

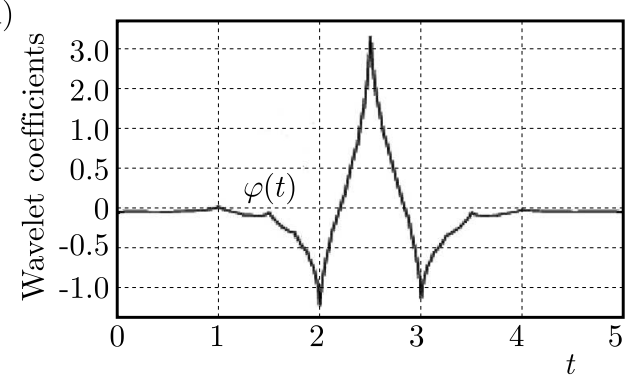

Fig. 2. Basis function (mother): (a) Daubechies 4, (c) Coiflet 6 wavelet and scaling function (father), (b) Daubechies 4 and (d) Coiflet 6 wavelet

and may be caused inter alia by measuring devices, measuring methods, measuring person, miscalculations or the influence of the environment on the previously mentioned causes. To investigate the efficency of DWT of a contaminated signal in damage detection and localization, measurement errors are accounted for by introduction of white noise to the output from computer simulated experiments. An example of randomly generated white noise is illustrated in Fig. 3.

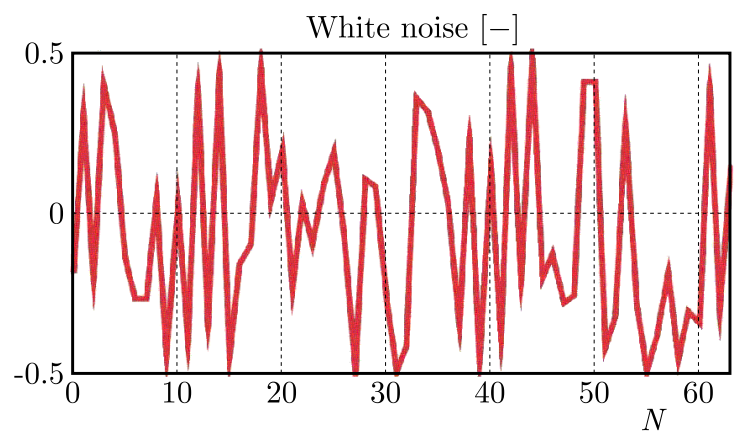

Fig. 3. White noise, values from -0.5 to 0.5

To adjust the size of the disturbance to the intensity of the analyzed response signal of the structure, white noise has been scaled by multiplying it by the constant number corresponding to the order of designated signal magnitude.

\section{Problem formulation of defect detection}

The aim of this work is to detect the localization of a defect provided that the defect (damage) exists in the considered plate structure. Numerical investigation is conducted being based on signal analysis of the structural static response. The plate material is assumed as linear-elastic. Plates are resting on elastic foundations: the Winkler type with bilateral and unilateral constraints and the elastic half-space with bilateral constraints. The plate bending is described and solved by the Boundary Element Method in the direct approach. The static fundamental solution (Green function) for a pure infinite plate is used (Guminiak, 2016) 


$$
w^{*}(r)=\frac{1}{8 \pi D} r^{2} \ln (r)
$$

which is a solution to the biharmonic equation

$$
\nabla^{4} w^{*}(r)=\frac{1}{D} \delta(r)
$$

for a thin isotropic plate, where $D=E h^{3} /\left[12\left(1-\nu^{2}\right)\right]$ is plate stiffness, $h$ is plate thickness, $E$ and $\nu$ are the Young modulus and Poisson ratio, $\delta(r)$ is the Dirac delta and $r=\sqrt{x^{2}+y^{2}}$.

\subsection{Modeling of the elastic internal support}

Mathematical description of behaviour of an elastic flexible foundation is usually given in form of an equation which connects reaction forces of the foundation $q_{0}$ with displacements $w$

$$
w(P)=\int_{\Omega_{0}} q_{0}(Q) g_{0}(P, Q) d \Omega_{0}
$$

For the simplest Winkler-type foundation in the discrete approach, a set of finite number of single independent and flexible supports is taken into account. Each of them has its stiffness $k$. For this type of foundation, the flexibility function has the following form

$$
g_{0}(P, Q)=\frac{1}{k} \delta(P, Q)
$$

where $\delta(P, Q)$ is the Dirac delta. After discretization, the Winkler-type foundation is described by the following diagonal stiffness matrix

$$
\mathbf{K}_{0}=\operatorname{diag}(k)
$$

The elastic half-space foundation with the elastic modulus $E_{0}$ and the Poisson ratio $\nu_{0}$ is considered. For this type of foundation, after its discretization using sub-domains of the constant type, the flexibility function has the form

$$
g_{0}(P, Q)=\frac{1-\nu_{0}^{2}}{\pi E_{0} r}
$$

and deformation of the foundation is expressed as

$$
w_{i}=\frac{1-\nu_{0}^{2}}{\pi E_{0}} \sum_{j=1}^{J} q_{0}(j) \int_{S_{j}} \frac{1}{r_{P Q}} d S_{j}
$$

In matrix notation, equation (4.7) will have the form

$$
\mathbf{w}_{0}=\mathbf{D}_{0} \mathbf{q}_{0}
$$

where $\mathbf{D}_{0}$ is the fully-populated flexibility matrix of the foundation. Using matrix equation (4.8), the reaction forces of the foundation can be obtained

$$
\mathbf{q}_{0}=\mathbf{K}_{0} \mathbf{w}_{0}
$$

where $\mathbf{K}_{0}=\mathbf{D}_{0}^{-1}$ is the fully-populated stiffness matrix of the foundation $\mathbf{K}_{0}=\left[k_{i j}\right]$.

The integral

$$
\int_{S_{j}} \frac{1}{r_{P Q}} d S_{j}
$$

can be calculated analytically (Fig. 4)

$$
\int_{\Omega_{0}} \frac{1}{r} d \Omega_{0}=x_{p} \ln \left(\frac{y_{p}+r_{1}}{y_{k}+r_{4}}\right)-y_{k} \ln \left(\frac{x_{p}+r_{4}}{x_{k}+r_{3}}\right)+x_{k} \ln \left(\frac{y_{k}+r_{3}}{y_{p}+r_{2}}\right)-y_{p} \ln \left(\frac{x_{k}+r_{2}}{x_{p}+r_{1}}\right)
$$




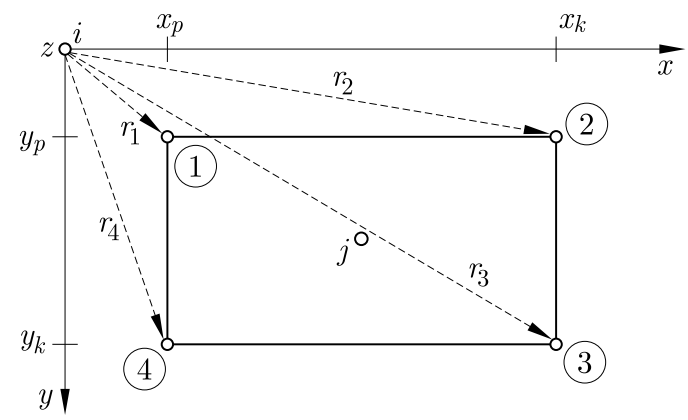

Fig. 4. Analytical calculation of integral (4.10) - assumed designations

\subsection{Boundary and domain integral equations}

Let the reaction forces of the foundation be realized as: a) set of concentrated forces $R_{0}^{(j)}$ or b) forces $q_{0}(j)$ distributed over the plate sub-domains. The Bézine technique (Bézine and Gamby, 1978) is introduced to derive the boundary-domain integral equations

$$
\begin{aligned}
& c(\mathbf{x}) w(\mathbf{x})+\int_{\Gamma}\left[T_{n}^{*}(\mathbf{y}, \mathbf{x}) w(\mathbf{y})-M_{n s}^{*}(\mathbf{y}, \mathbf{x}) \frac{d w(\mathbf{y})}{d s}-M_{n}^{*}(\mathbf{y}, \mathbf{x}) \varphi_{n}(\mathbf{y})\right] d \Gamma(\mathbf{y}) \\
& =\int_{\Gamma}\left[\widetilde{T}_{n}(\mathbf{y}) w^{*}(\mathbf{y}, \mathbf{x})-M_{n}(\mathbf{y}) \varphi_{n}^{*}(\mathbf{y}, \mathbf{x})\right] d \Gamma(\mathbf{y})-\sum_{j=1}^{J} R_{0}^{(j)} w^{*}(j, \mathbf{x})+\int_{\Omega} p(\mathbf{y}) w^{*}(\mathbf{y}, \mathbf{x}) d \Omega(\mathbf{y}) \\
& c(\mathbf{x}) \varphi_{n}(\mathbf{x})+\int_{\Gamma}\left[\bar{T}_{n}^{*}(\mathbf{y}, \mathbf{x}) w(\mathbf{y})-\bar{M}_{n s}^{*}(\mathbf{y}, \mathbf{x}) \frac{d w(\mathbf{y})}{d s}-\bar{M}_{n}^{*}(\mathbf{y}, \mathbf{x}) \varphi_{n}(\mathbf{y})\right] d \Gamma(\mathbf{y}) \\
& =\int_{\Gamma}\left[\widetilde{T}_{n}(\mathbf{y}) \bar{w}^{*}(\mathbf{y}, \mathbf{x})-M_{n}(\mathbf{y}) \bar{\varphi}_{n}^{*}(\mathbf{y}, \mathbf{x})\right] d \Gamma(\mathbf{y})-\sum_{j=1}^{J} R_{0}^{(j)} \bar{w}^{*}(j, \mathbf{x})+\int_{\Omega} p(\mathbf{y}) \bar{w}^{*}(\mathbf{y}, \mathbf{x}) d \Omega(\mathbf{y})
\end{aligned}
$$

for case a) and

$$
\begin{aligned}
& c(\mathbf{x}) w(\mathbf{x})+\int_{\Gamma}\left[T_{n}^{*}(\mathbf{y}, \mathbf{x}) w(\mathbf{y})-M_{n s}^{*}(\mathbf{y}, \mathbf{x}) \frac{d w(\mathbf{y})}{d s}-M_{n}^{*}(\mathbf{y}, \mathbf{x}) \varphi_{n}(\mathbf{y})\right] d \Gamma(\mathbf{y}) \\
&= \int_{\Gamma}\left[\widetilde{T}_{n}(\mathbf{y}) w^{*}(\mathbf{y}, \mathbf{x})-M_{n}(\mathbf{y}) \varphi_{n}^{*}(\mathbf{y}, \mathbf{x})\right] d \Gamma(\mathbf{y}) \\
&- \sum_{j=1}^{J}\left(\int_{\Omega_{0}(j)} q_{0}(j) w^{*}(j, \mathbf{x}) d \Omega_{0}(j)\right)+\int_{\Omega} p(\mathbf{y}) w^{*}(\mathbf{y}, \mathbf{x}) d \Omega(\mathbf{y}) \\
& c(\mathbf{x}) \varphi_{n}(\mathbf{x})+\int_{\Gamma}\left[\bar{T}_{n}^{*}(\mathbf{y}, \mathbf{x}) w(\mathbf{y})-\bar{M}_{n s}^{*}(\mathbf{y}, \mathbf{x}) \frac{d w(\mathbf{y})}{d s}-\bar{M}_{n}^{*}(\mathbf{y}, \mathbf{x}) \varphi_{n}(\mathbf{y})\right] d \Gamma(\mathbf{y}) \\
&=\int_{\Gamma}\left[\widetilde{T}_{n}(\mathbf{y}) \bar{w}^{*}(\mathbf{y}, \mathbf{x})-M_{n}(\mathbf{y}) \bar{\varphi}_{n}^{*}(\mathbf{y}, \mathbf{x})\right] d \Gamma(\mathbf{y}) \\
&-\sum_{j=1}^{J}\left(\int_{\Omega_{0}(j)} q_{0}(j) \bar{w}^{*}(j, \mathbf{x}) d \Omega_{0}(j)\right)+\int_{\Omega} p(\mathbf{y}) \bar{w}^{*}(\mathbf{y}, \mathbf{x}) d \Omega(\mathbf{y})
\end{aligned}
$$

for case $\mathrm{b}$ ), where $\mathbf{x}$ is the source point, $\mathbf{y}$ is the field point and $r=|\mathbf{y}-\mathbf{x}|$. The second boundary-domain integral equations $(4.12)_{2}$ for case a) and $(4.13)_{2}$ for case b) can be obtained replacing the unit concentrated force $P^{*}=1$ by the unit concentrated moment $M_{n}^{*}=1$. Such 
a replacement is equivalent to the differentiation of the first boundary integral equation $(4.12)_{1}$ or $(4.13)_{1}$ with respect to the coordinate $n$ at a point $\mathbf{x}$ belonging to the plate domain, and letting this point approach the boundary and taking $n$ coincide with the normal to it. The force $\widetilde{T}_{n}(\mathbf{y})$ can be treated as an equivalent shear force $V_{n}(\mathbf{y})$ on a fragment of the boundary which is located far from the corner, or it plays the role of the corner force $R_{n}(\mathbf{y})$ which is distributed on a small fragment of the boundary close to the corner. In the case of the free edge, we must combine the rotation angle in the tangent direction $\varphi_{s}(\mathbf{y})$ with the fundamental function $M_{n s}^{*}(\mathbf{y})$. Since the relation between $\varphi_{s}(\mathbf{y})$ and the deflection is known: $\varphi_{s}(\mathbf{y})=d w(\mathbf{y}) / d s$, the angle of rotation $\varphi_{s}(\mathbf{y})$ can be evaluated while using a finite difference scheme of the deflection with two or more adjacent nodal values. In this analysis, the employed finite difference scheme includes the deflections of two adjacent nodes.

\subsection{Construction of the set of algebraic equation}

The set of algebraic equations in matrix notation has the following form

$$
\left[\begin{array}{ccc}
\mathbf{G}_{B B} & \mathbf{G}_{B S} & \mathbf{G}_{B w} \mathbf{K}_{0} \\
\boldsymbol{\Delta} & -\mathbf{I} & \mathbf{0} \\
\mathbf{G}_{w B} & \mathbf{G}_{w S} & \mathbf{G}_{w w} \mathbf{K}_{0}
\end{array}\right]\left\{\begin{array}{c}
\mathbf{B} \\
\boldsymbol{\varphi}_{s} \\
\mathbf{w}
\end{array}\right\}=\left\{\begin{array}{c}
\mathbf{F}_{B} \\
\mathbf{0} \\
\mathbf{F}_{R}
\end{array}\right\}
$$
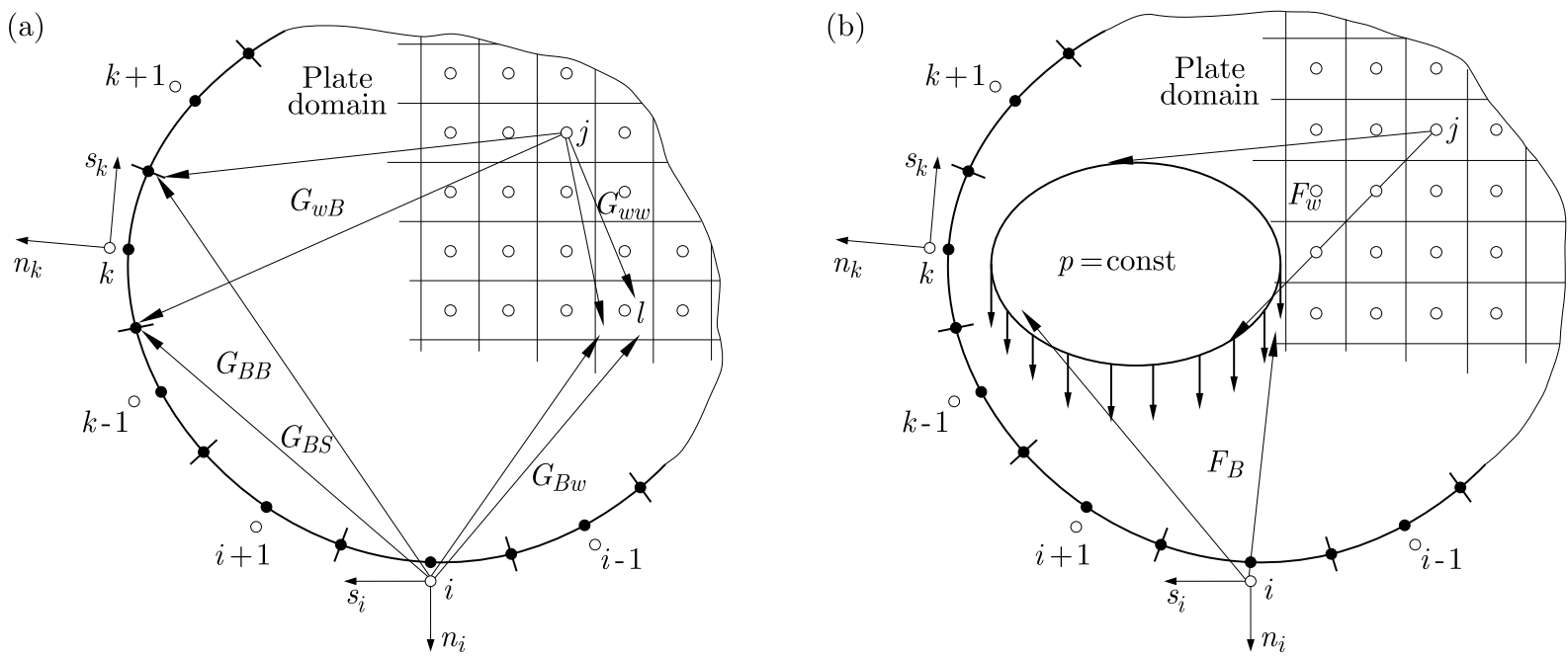

Fig. 5. Construction of the characteristic matrix

Suitable designations of sub-matrices occurring in matrix equation (4.14) are presented in Fig. 5 for (a) characteristic matrix and (b) right-hand-side vector, where: $\mathbf{G}_{B B}$ and $\mathbf{G}_{B S}$ are the matrices of dimension $(2 N \times 2 N)$ and of dimension $(2 N \times S)$ grouping boundary integrals and depending on the type of the boundary, where $N$ is the number of boundary nodes (or the number of constant type elements). $S$ is the number of boundary elements along the free edge; $\mathbf{G}_{B w}$ is the matrix of dimension $(2 N \times M)$ grouping values of the fundamental function $w^{*}$ established at the internal collocation points; $\boldsymbol{\Delta}$ is the matrix grouping difference operators connecting the angle of rotations in the tangent direction with deflections of suitable boundary nodes if the plate has a free edge; $\mathbf{G}_{w B}$ is the matrix of dimension $(M \times 2 N)$ grouping the boundary integrals of the appropriate fundamental functions, where $M$ is the number of the internal collocation points and $N$ is the number of the boundary nodes; $\mathbf{G}_{w S}$ is the matrix of dimension $(M \times S)$ grouping the boundary integrals of the appropriate fundamental functions; $\mathbf{G}_{w w}$ is the matrix of dimension $(M \times M)$ grouping the values of the fundamental function $w^{*}$ established at the internal collocation points; $\mathbf{F}_{B}$ is the right-hand side vector in which all 
elements are calculated in the coordinate systems connected with the boundary physical nodes over the loading area; $\mathbf{F}_{w}$ is the right-hand side vector whose all elements are calculated in the coordinate systems connected with the internal collocation points over the loading area; $\mathbf{K}_{0}$ is the stiffness matrix of the foundation.

\subsection{Calculation of deflection and internal forces inside the plate domain}

The solution of the set of equations (4.14) allows one to determine suitable boundary variables and values of deflection at the internal collocation points associated with flexible constraints. Then, using the same boundary and domain integral equation $(4.12)_{1}$ or $(4.13)_{1}$, deflection at an arbitrary point of plate domain can be calculated. The collocation point is located inside the plate domain, hence the coefficient $c(\mathbf{x})$ is equal to one. Plate deflection can be expressed as the sum of the deflection depending on boundary variables, the deflections (or reactions) at the internal collocation points and the external loading, respectively

$$
w=w(\overline{\mathbf{B}})+w(\mathbf{w})+w(p)
$$

To calculate deflection at a selected point, equations $(4.12)_{1}$ or $(4.13)_{1}$ can be directly used for Winkler and elastic half-space type of foundations, respectively.

\section{Numerical examples}

Rectangular plate structures with free boundaries are considered. Defects are introduced by additional edges forming slots or holes in relation to the basic plate domain. The Boundary Element Method is applied to solve a thin plate bending problem. Each plate edge is divided into 30 boundary elements of the constant type. The collocation point is located slightly outside the plate edge, which is estimated by the parameter $\varepsilon=\bar{\delta} / d$, where $\delta$ is the real distance of the collocation point from the plate edge and $d$ is the element length. For each example, it is assumed $\varepsilon=0.001$. Diagonal boundary terms in the characteristic matrix are calculated analytically and the rest of them use 12-point Gauss quadrature. Plate properties are $E=205.0 \mathrm{GPa}, \nu=0.3$, $h=0.04 \mathrm{~m}$. The plates are loaded statically. The static concentrated external load $P=10.0 \mathrm{kN}$ which is moving along the line parallel to the global coordinate $x$ is replaced by an equivalent uniformly distributed loading $p$ acting over the square surface of dimensions $0.05 \mathrm{~m} \times 0.05 \mathrm{~m}$. Except for that, plates subjected only to a uniformly distributed loading $q=10.0 \mathrm{kN} / \mathrm{m}^{2}$ acting on the surface are considered too. The examined plates are resting on the Winkler and elastic half-space types of foundation. The stiffness coefficient of the Winkler foundation is $k=5000.0 \mathrm{kN} / \mathrm{m}^{3}$ and the properties of the elastic half-space are $E_{0}=30000 \mathrm{kN} / \mathrm{m}^{2}, \nu_{0}=0.4$. As a structural response, the influence line of deflections and vertical displacements are taken into account. The data is gathered in one measurement point in equal time intervals or in 64 points. Decomposition of the obtained signal is carried out using DWT with Daubechies 4, Coiflet 6 and Coiflet 12 families of wavelets. For the selected example, white noise is introduced too.

\subsection{Example 1}

The plate resting on the Winkler foundation loaded by a static concentrated force $P$ which is moving along the edge parallel to the $x$ direction is considered and presented in Fig. 6 . The measurement point $D$ has the following coordinates: $x_{D}=2.2 \mathrm{~m}$ and $y_{D}=0.2 \mathrm{~m}$. The introduced plate defect is described by the parameter: $e=0.005 \mathrm{~m}$.

The results of calculation for bilateral constraints are presented in Fig. 7 for (a) Daubechies 4 and (b) Coiflet 12 families of wavelets, respectively. Detail 1 of the decomposed response signal is analysed. It is visible that in both cases the damage location is clearly depicted by the evident 


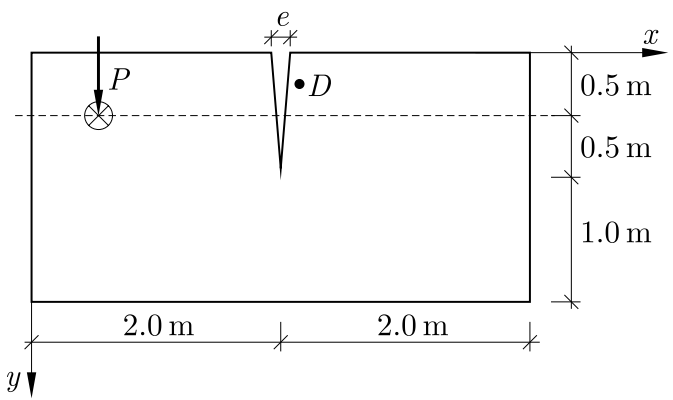

Fig. 6. Considered plate structure

disturbances and high picks. Nonetheless, noteworthy is the fact that in Coiflet 12 DWT, the transformed signal is smoother and the boundary disturbances in transformation window are slightly smaller (Fig. 7b).

(a)

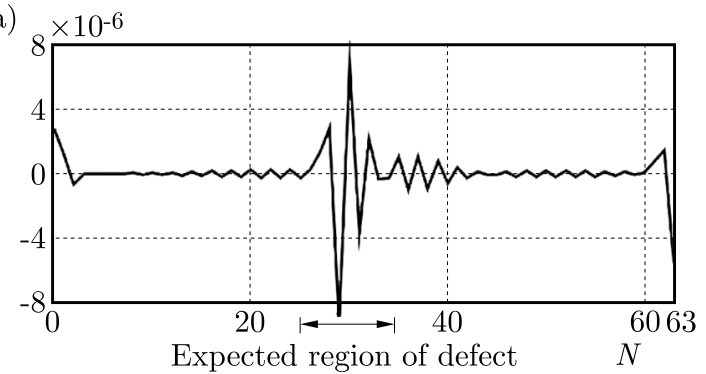

(b)

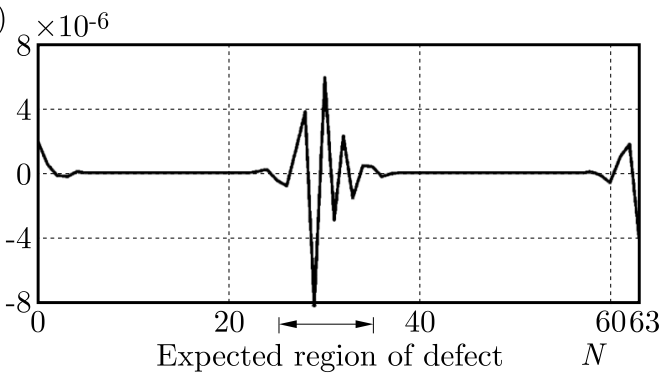

Fig. 7. DWT (Daubechies 4, Coiflet 12, detail 1) signal: vertical displacements measured at the point $D$, $N$ - number of measurements

The results of calculation for unilateral constraints are presented in Fig. 8 for (a) Daubechies 4 and (b) Coiflet 12 families of wavelets, respectively. Detail 1 of the decomposed response signal has been analysed. However, in this analysis, expected disturbances of the transformed signal do not properly indicate the damage location. The highest pick is unfortunately elsewhere. Presumably, at a finite number of iterations with exclusion of nodes in which the reaction force is negative, the problem formulation in terms of the BEM causes bad conditioning of the characteristic matrix.
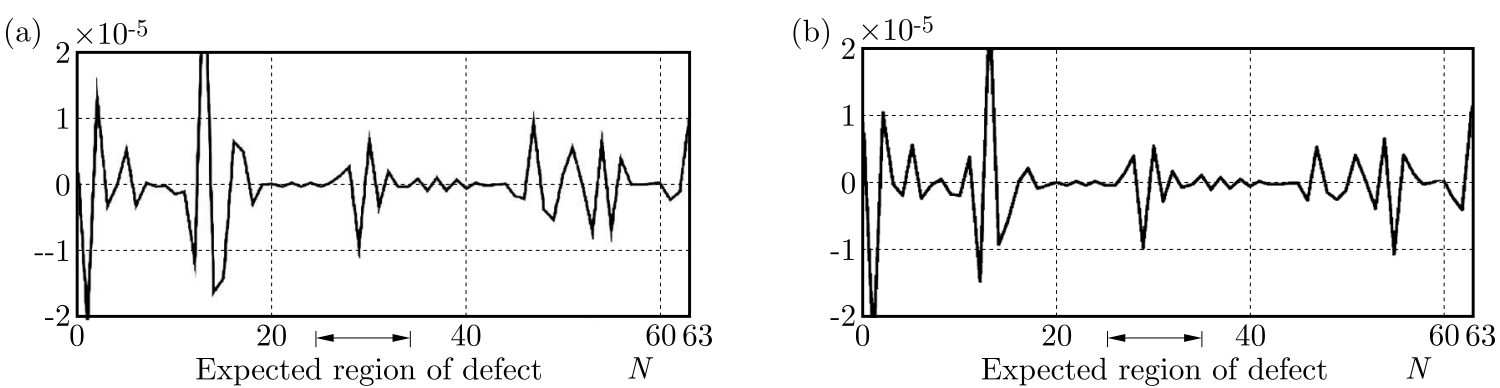

Fig. 8. DWT (Daubechies 4, Coiflet 12, detail 1) signal: vertical displacements measured at the point $D$, $N$ - number of measurements

\subsection{Example 2}

The plate resting on the Winkler foundation with bilateral constrains and loaded by a static uniformly distribute loading $q$ is considered and presented in Figs. 9 and 12. Damage is identified by the parameter $e=0.005 \mathrm{~m}$. 
a) External loading $q$ is acting on the square whose corners $(A, B, C$ and $D)$ have coordinates $x$ and $y$, respectively (Fig. 9): $A(0.6 \mathrm{~m} ; 0.6 \mathrm{~m}), B(1.4 \mathrm{~m} ; 0.6 \mathrm{~m}), C(0.6 \mathrm{~m} ; 1.4 \mathrm{~m})$ and $D(1.4 \mathrm{~m} ; 1.4 \mathrm{~m})$.

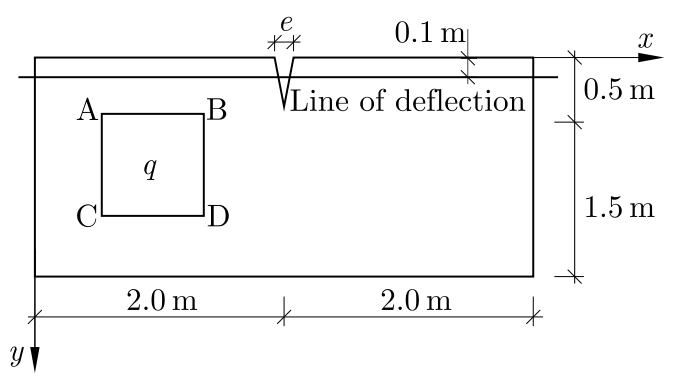

Fig. 9. Considered plate structure

The results of calculation are presented in Fig. 10 for (a) Daubechies 4 and (b) Coiflet 6 families of wavelets, respectively. Detail 1 of the decomposed response signal has been analysed. The damage is undoubtedly located properly in both transforms by a high pick.

(a)

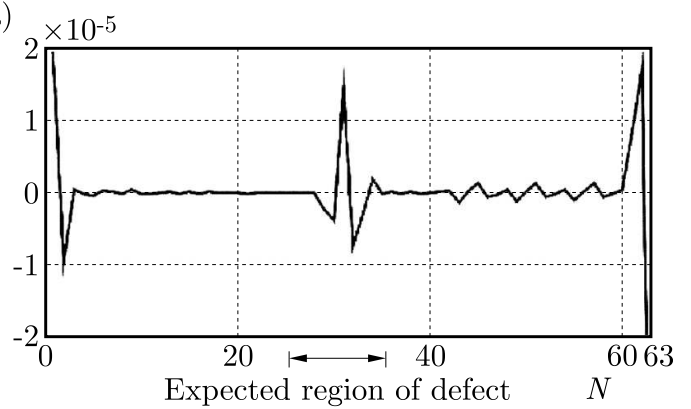

(b)

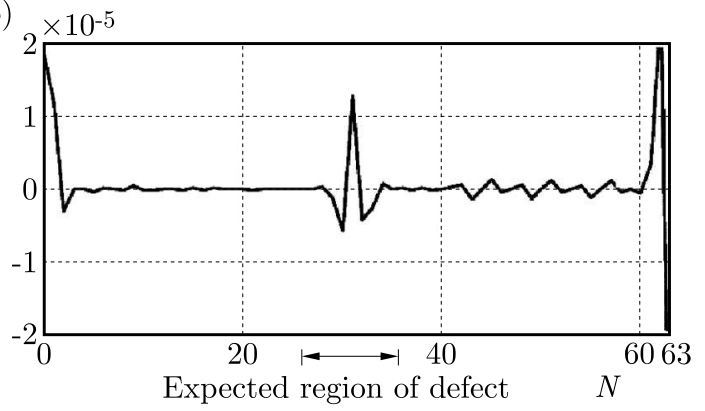

Fig. 10. DWT (Daubechies 4, Coiflet 6, detail 1) signal: vertical displacements, $N$ - number of measurement points

In this example, randomly generated white noise has been introduced. The maximum intensity of the introduced disturbance has $5 \%$ of the response signal measured value (relative to the highest measured value). The results of calculation are presented in Fig. 11 for (a) Daubechies 4 and (b) Coiflet 6 families of wavelets respectively. The DWT (detail 1) of the vertical displacements has been analysed. In both cases, the damage is properly localized by a high pick, however, previous experiments show that $5 \%$ noise in the transformed data is for DWT the upper limit of damage detection capability. While considering more contaminated signals, one can apply signal denoising techniques, e.g. the wavelet shrinkage method (Dobrzycki and Mikulski, 2016).
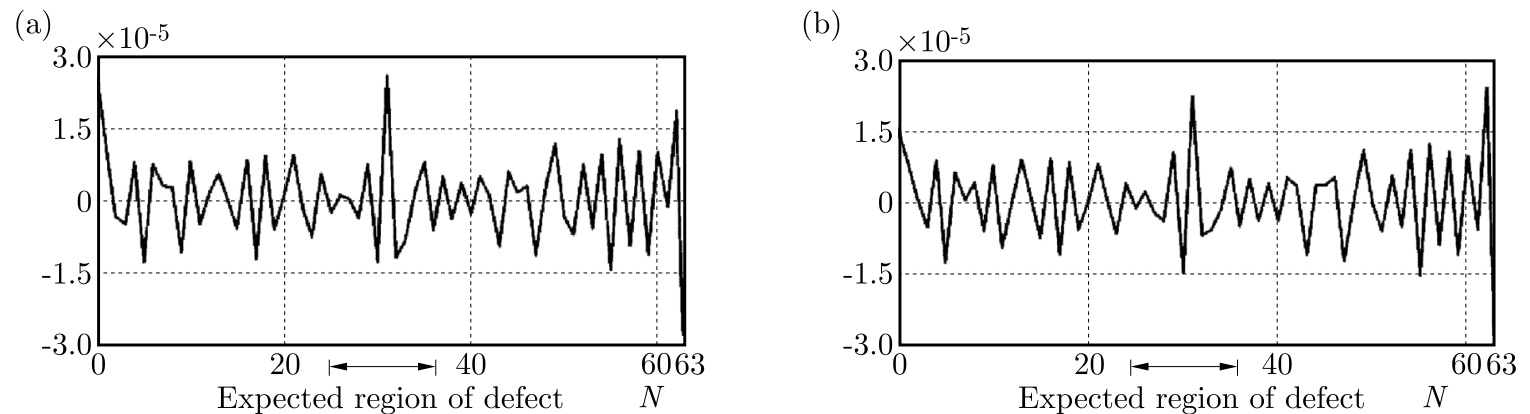

Fig. 11. DWT (Daubechies 4, Coiflet 6, detail 1) signal: vertical displacements, $N$-number of measurement points 
b) External loading $q$ is acting on the square whose corners $(A, B, C$ and $D)$ have coordinates $x$ and $y$, respectively (Fig. 12): $A(2.6 \mathrm{~m} ; 0.2 \mathrm{~m}), B(3.4 \mathrm{~m} ; 0.2 \mathrm{~m}), C(2.6 \mathrm{~m} ; 1.0 \mathrm{~m})$ and $D(3.4 \mathrm{~m} ; 1.0 \mathrm{~m})$.

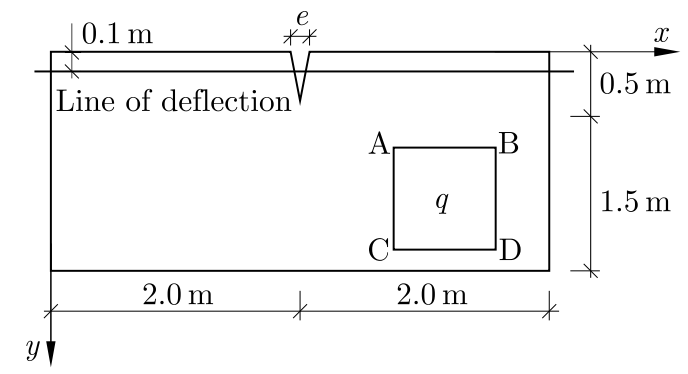

Fig. 12. Considered plate structure

The results of calculation are presented in Fig. 13 for (a) Daubechies 4 and (b) Coiflet 6 families of wavelets respectively. Detail 1 of the transformed vertical displacements signal is shown. In both cases, the damage existence and location are properly detected.

(a)

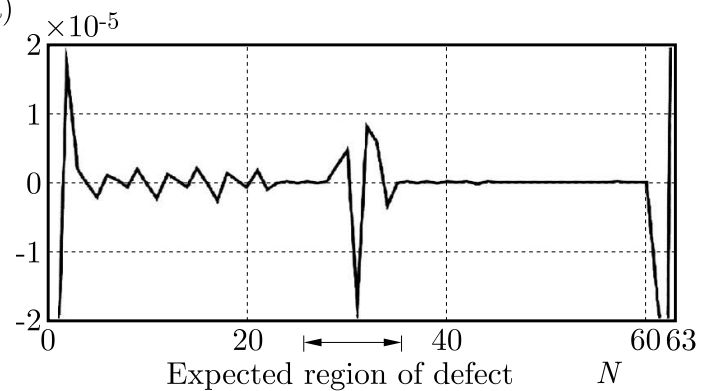

(b)

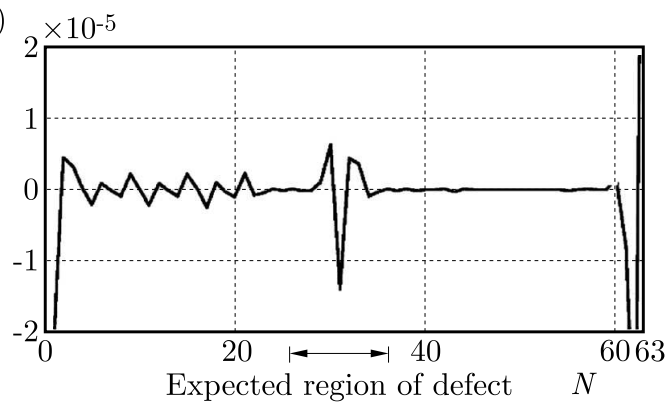

Fig. 13. DWT (Daubechies 4, Coiflet 6, detail 1) signal: vertical displacements, $N$ - number of measurement points

\subsection{Example 3}

The plate resting on the elastic half-space foundation, loaded by a static concentrated force $P$ which is moving along the edge parallel to the $x$ direction is considered. The plate geometry and loading is presented in Fig. 14. The measurement point $D$ has following coordinates: $x_{D}=2.2 \mathrm{~m}$ and $y_{D}=0.2 \mathrm{~m}$. The introduced plate defect is described by the parameter: $e=0.01 \mathrm{~m}$. Bilateral constraints are assumed in the analysis. The results of calculation are presented in Fig. 15 for (a) Daubechies 4 and (b) Coiflet 12 wavelet families, respectively. The DWT (detail 1) of the vertical displacements influence line is shown. The damage location is clearly depicted by the evident disturbances of the transformed data in both cases. It is worth to emphasize that while using Coiflet 12 DWT, the transformed signal is smoother and the disturbance region is slightly shorter (Fig. 15b).

\subsection{Example 4}

The plate resting on the elastic half-space foundation with bilateral constrains and loaded by a static uniformly distribute loading $q$ is considered. The plate geometry is the same as presented in Figs. 12 and 16. The damage is identified by the parameter $e=0.005 \mathrm{~m}$. The analysis has been carried out for the bilateral constraints.

a) External loading $q$ is acting on the square whose corners $(A, B, C$ and $D)$ have coordinates $x$ and $y$, respectively (Fig. 16): $A(2.6 \mathrm{~m} ; 0.2 \mathrm{~m}), B(3.4 \mathrm{~m} ; 0.2 \mathrm{~m}), C(2.6 \mathrm{~m} ; 1.0 \mathrm{~m})$ and $D(3.4 \mathrm{~m} ; 1.0 \mathrm{~m})$. The results of DWT (detail 1) of the vertical displacement signals measured in 64 points are 


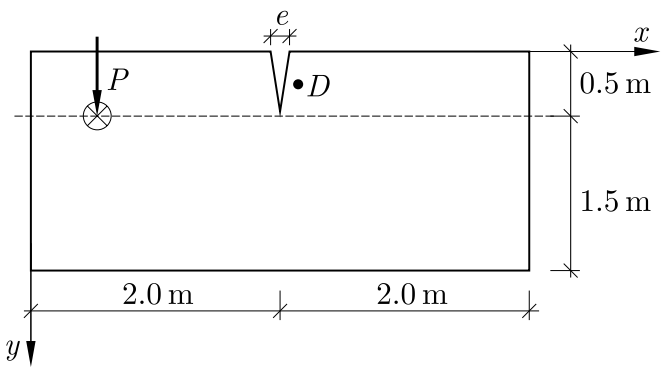

Fig. 14. Considered plate structure
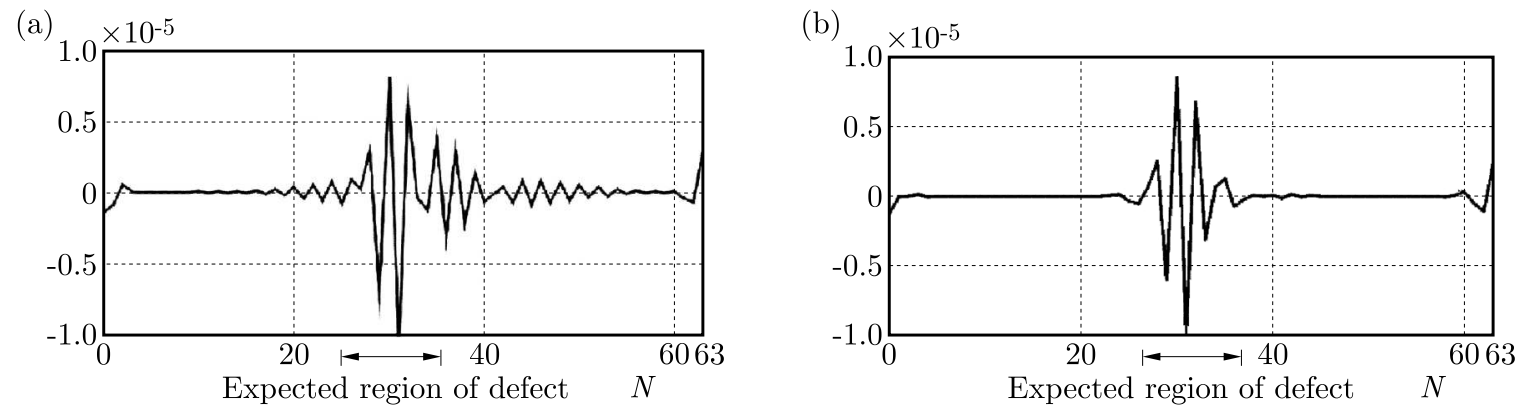

Fig. 15. DWT (Daubechies 4, Coiflet 12, detail 1) signal: vertical displacements measured at the point $D, N$ - number of measurements

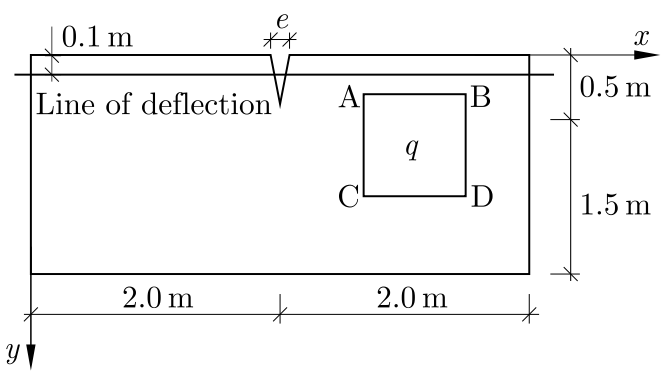

Fig. 16. Considered plate structure

(a)

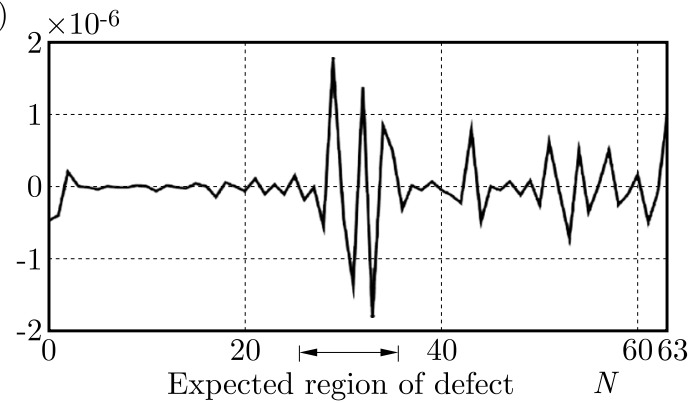

(b)

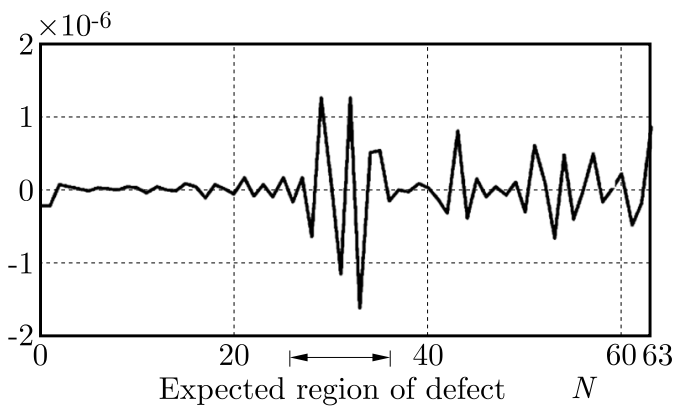

Fig. 17. DWT (Daubechies 4, Coiflet 12, detail 1) signal: vertical displacements, $N$ - number of measurement points

presented in Fig. 17 for (a) Daubechies 4 and (b) Coiflet 12 wavelets. The damage location can be clearly determined by high picks in both cases despite quite large disturbances on the right hand side in the windows of transformation.

b) External loading $q$ is acting on the square whose corners $(A, B, C$ and $D)$ have coordinates $x$ and $y$, respectively (Fig. 12): $A(2.8 \mathrm{~m} ; 1.0 \mathrm{~m}), B(3.6 \mathrm{~m} ; 1.0 \mathrm{~m}), C(2.8 \mathrm{~m} ; 1.8 \mathrm{~m})$ and $D(3.6 \mathrm{~m} ; 1.8 \mathrm{~m})$. The results of DWT (detail 1) of the vertical displacement signals measured in 64 points are 
presented in Fig. 18 for (a) Daubechies 4 and (b) Coiflet 6 families of wavelets, respectively. In both cases, the damage existence and location are properly detected by high picks of the transformed signal.

(a)

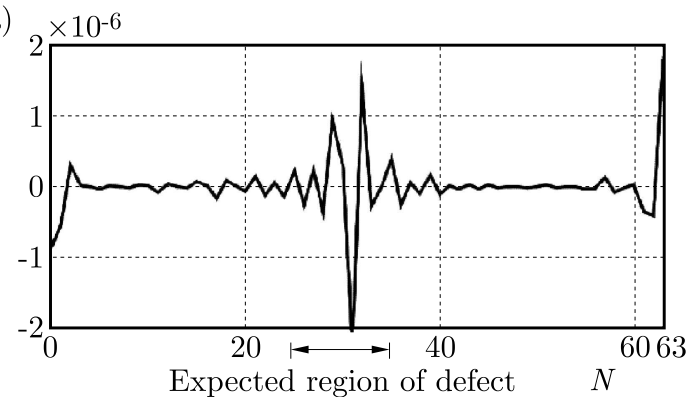

(b)

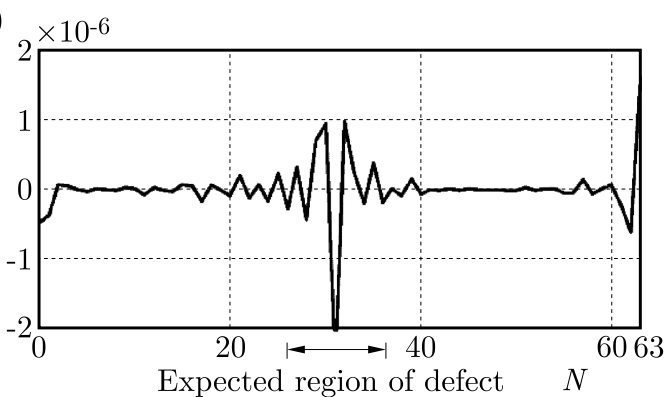

Fig. 18. DWT (Daubechies 4, Coiflet 6, detail 1) signal: vertical displacements measured along the line of deflection, $N$ - number of measurement point

\section{Concluding remarks}

The implementation of a discrete dyadic wavelet transformation to identification of signal discontinuity in the analysis of plates resting on internal flexible supports is presented in the this paper. The novelty is the introduction of defects by additional edges forming slots or holes in the relation to the basic plate domain. Thin (Kirchhoff) plate bending is described by boundary-domain integral equations and solved using the Boundary Element Method (BEM). The BEM is particularly useful to carry out a numerical experiment of plate bending considering abnormal shapes of the plate edge having the character of cracks or tears. Although the considered issue is two-dimensional from the point of view of deformation description, the applied one-dimensional DWT leads to efficient results in defect detection. The proposed method does not provide detection of defects localized inside the plate domain. The measured response signal is assumed as the influence line of deflection considered at a selected point and the deflection line. The minimum number of measurements has been assumed as sixty four. The damage is properly localized while using asymmetric Daubechies 4 wavelet as well as nearly symmetrical Coiflet 6 or 12 wavelet in signal decomposition. Detail 1, as the most detailed representation of the transformed signal, is taken into consideration. The efficiency of DWT in damage detection and localization does not depend on the position of the uniformly distributed load, yet the distance of the measurement point from the damaged area in the case of the influence line of deflection is crucial. White noise as a innacuracy of the measured signal has also been considered in the selected example. The position of defects has been quite correctly identified by a high pick of the transformed (both Daubechies and Coiflet wavelet) data, however it should be noted that $5 \%$ noise in the transformed data is for DWT the upper limit of damage detection capability. While considering more contaminated signals, one can apply signal denoising techniques, e.g. the wavelet shrinkage method.

\section{References}

1. BÉzine G., Gamby D.A., 1978, A new integral equations formulation for plate bending problems, Advances in Boundary Element Method, Pentech Press, London

2. Dems K., Mróz, Z., 2001, Identification of damage in beam and plate structures using parameterdependent frequency changes, Engineering Computations, 18, 1/2, 96-120 
3. Dobrzycki A., Mikulski S., 2016, Using of continuous wavelet transform for de-noising signals accompanying electrical treeing in epoxy resins, Przeglad Elektrotechniczny (Electrotechnical Review), 92, 4, 26-29, DOI: 10.15199/48.2016.04.07

4. Dodge Y., 2003, The Oxford Dictionary of Statistical Terms, Oxford University Press

5. Garbowski T., Maier G., Novati G., 2011, Diagnosis of concrete dams by flat-jack tests and inverse analysis based on proper othogonal decomposition, Journal of Mechanics of Materials and Structures, 6, 1-4, 181-202

6. Guminiak M., 2016, Static and free vibration analysis of thin plates of the curved edges by the boundary element method considering an alternative formulation of boundary conditions, Engineering Transactions, 64, 1, 3-32

7. Knitter-Piatkowska A., Garbowski, T., 2013, Damage detection through wavelet transform and inverse analysis, VI International Conference on Adaptive Modelling and Simulation (ADMOS 2013), J. Moitinho de Almeida, P. Diez, C. Tiago, and N. Pares (Edit.), Barcelona, 389-400

8. Knitter-Piątkowska A., Guminiak M., Hloupis G., 2017, Crack identification in plates using 1-D discrete wavelet transform, Journal of Theoretical and Applied Mechanics, 55, 2, 481-496

9. Knitter-Piątrowska A., Guminiak M., Przychodzki M., 2014, Damage Detection in Truss Structure Being the Part of Historic Railway Viaduct Wavelet Transformation, T. Łodygowski, J. Rakowski and P. Litewka (Edit.), CRC Press/Balkema, Taylor and Francis Group

10. Mallat S.G., 1989, A theory for multiresolution signal decomposition: the wavelet representation, IEEE Transactions on Pattern Analysis and Machine Intelligence, 11, 7, 674-693

11. Mallat S.G., 1999, A Wavelet Tour of Signal Processing, Academic Press, San Diego

12. Mróz Z., Garstecki A., 2005, Optimal loading conditions in design and identification of structures. Part 1: Discrete formulation, International Journal of Structural and Multidisciplinary Optimization, 29, 11-18

13. WANG Q., Deng X., 1999, Damage detection with spatial wavelets, International Journal of Solids and Structures, 36, 3443-3448

14. Ziopaja K., Pozorski Z., Garstecki A., 2011, Damage detection using thermal experiments and wavelet transformation, Inverse Problems in Science and Engineering, 19, 1, 127-153 